\title{
A COMPARISON OF GRAIN SIZE MEASUREMENTS IN AL-CU THIN FILMS: IMAGING VERSES DIFFRACTION TECHNIQUES
}

\author{
L.M. Gignac, ${ }^{*}$ C.E. Murray, ${ }^{*}$ K.P. Rodbell, ${ }^{*}$ M. Gribelyuk ${ }^{+}$ \\ *IBM T.J. Watson Research Center, Yorktown Heights, NY 10598 \\ +IBM Microelectronics Division, Hopewell Junction, NY 12533
}

Numerous microscopy techniques, based on both imaging and diffraction, exist for the measurement of grain size distributions in polycrystalline thin-film samples. The accuracy of each technique is affected by two major factors: the effective resolution of the instrument relative to the characteristic grain size and the recognition of boundaries between adjacent grains during postprocessing. Techniques sensitive to the sample surface, such as focused ion beam (FIB) microscopy and atomic force microscopy (AFM), can be used under the assumption that the thin film possess a columnar microstructure. Electron backscatter patterning (EBSP) is sensitive to the grain orientation within approximately $50 \mathrm{~nm}$ below the thin film surface whereas scanning transmission electron microscopy (STEM) obtains information through the thin film thickness. Several techniques rely on manual or automated recognition of grain boundaries through differential contrast between the grains (STEM, FIB) or at grain boundaries (AFM). EBSP delineates grains by calculating the relative misorientation of adjacent data points. In this study, we compared the effectiveness of these techniques in determining grain size information for several sputter-deposited thin films.

Grain area distributions were measured for a series of three $0.5 \mu \mathrm{m}$ thick Al-0.5wt. $\% \mathrm{Cu}$ blanket thin films deposited on either $\mathrm{Si} / \mathrm{SiN}_{\mathrm{x}}$ or $\mathrm{Si} /$ phosphous-silicate glass PSG substrates. These films had median grain sizes ranging from $0.5-2.5 \mu \mathrm{m}$. The thin film microstructure was first measured using various grain imaging techniques including FIB, STEM, and AFM. For each of these methods, images of the grain structures were obtained, the grains were hand traced and then digitized, the grain areas were measured, and log-normal distributions of the grain areas were plotted. Median grain diameters were determined from the median grain areas assuming circular grains. The grain area distributions derived from these imaging techniques were compared to area distributions determined from EBSP orientation imaging where grains boundaries were defined as greater than 10 degrees of misorientation between adjacent EBSP patterns. FIG. 1a-c show a comparison of grain structure images obtained by AFM, FIB microscopy and EBSP orientation imaging, respectively, taken at similar magnifications for a $0.5 \mu \mathrm{m}$ thick Al sample which had a median grain diameter of $\sim 2 \mu \mathrm{m}$. FIG. 2 shows a comparison of the median grain areas and corresponding median grain diameters for three samples which have median grain diameters of $\sim 2$ $\mu \mathrm{m}, \sim 1 \mu \mathrm{m}$ and $\sim 0.5 \mu \mathrm{m}$, respectively. For the film with a median grain diameter of $\sim 0.5 \mu \mathrm{m}$, there is significant variation in the median grain area measurements. An understanding of the accuracy of grain size and shape determination using EBSP orientation imaging was obtained by marking regions on these samples with the FIB and then EBSP imaging these identical regions. FIG. 3a,b are FIB and EBSP images, respectively, where both images were taken of the same region on the sample. FIG. $3 c$ is an overlay image of the grains traced from the FIB image (shown in white) on the EBSP image. These images show that for large grain areas, the EBSP orientation images determine similar grain morphology as FIB imaging but some of the small grains shown in the FIB images are not imaged as distinct grains in the EBSP image. The missing small grains in the EBSP images have been attributed to choosing too large of a step size during analysis and errors in pattern recognition. 

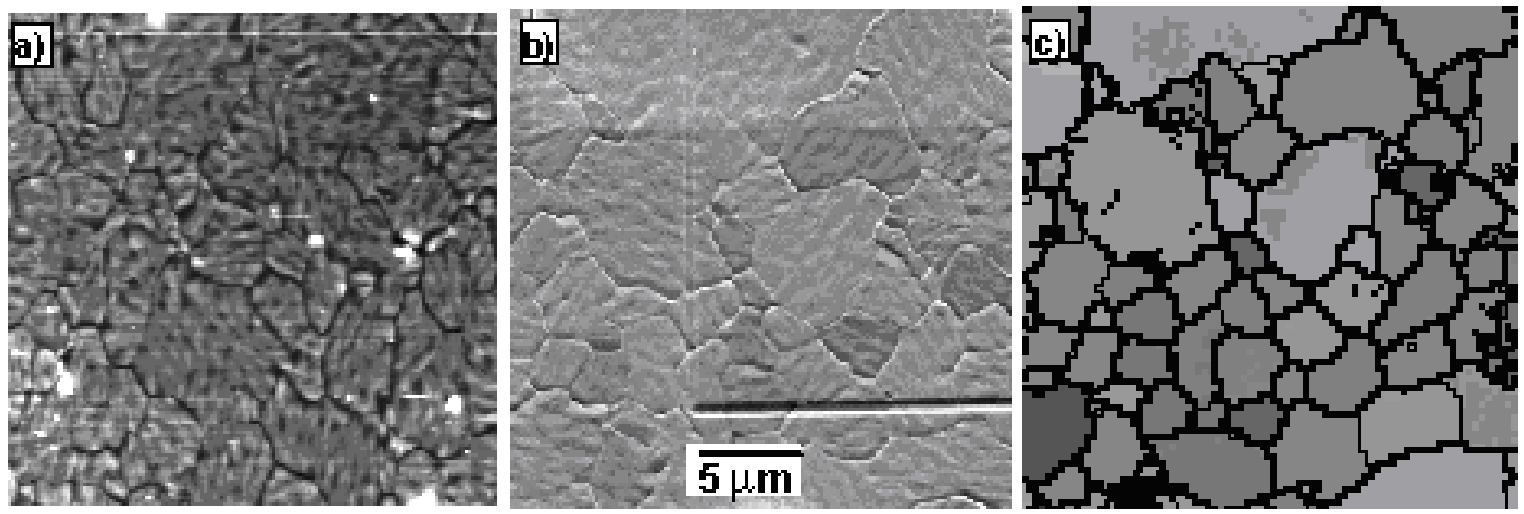

FIG. 1: Various microstructural images of an $\mathrm{Al}-0.5 \% \mathrm{Cu}$ blanket film on $\mathrm{Si}_{3} \mathrm{~N}_{4}$ with a median grain diameter of $\sim 2.0$ $\mu \mathrm{m}$ (sample 2N): a) AFM surface plot, b) FIB secondary electron image generated a from ion beam, and c) EBSP grain orientation image.

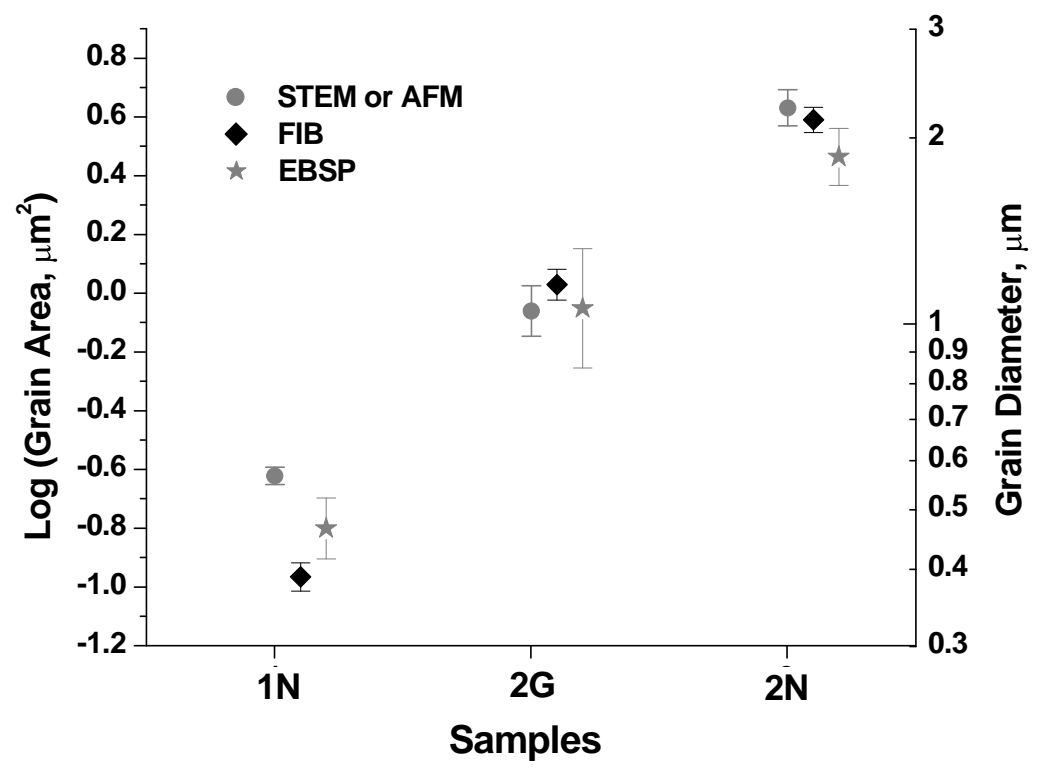

FIG. 2: Comparison of grain size data from STEM/AFM, FIB and EBSP measurement methods.
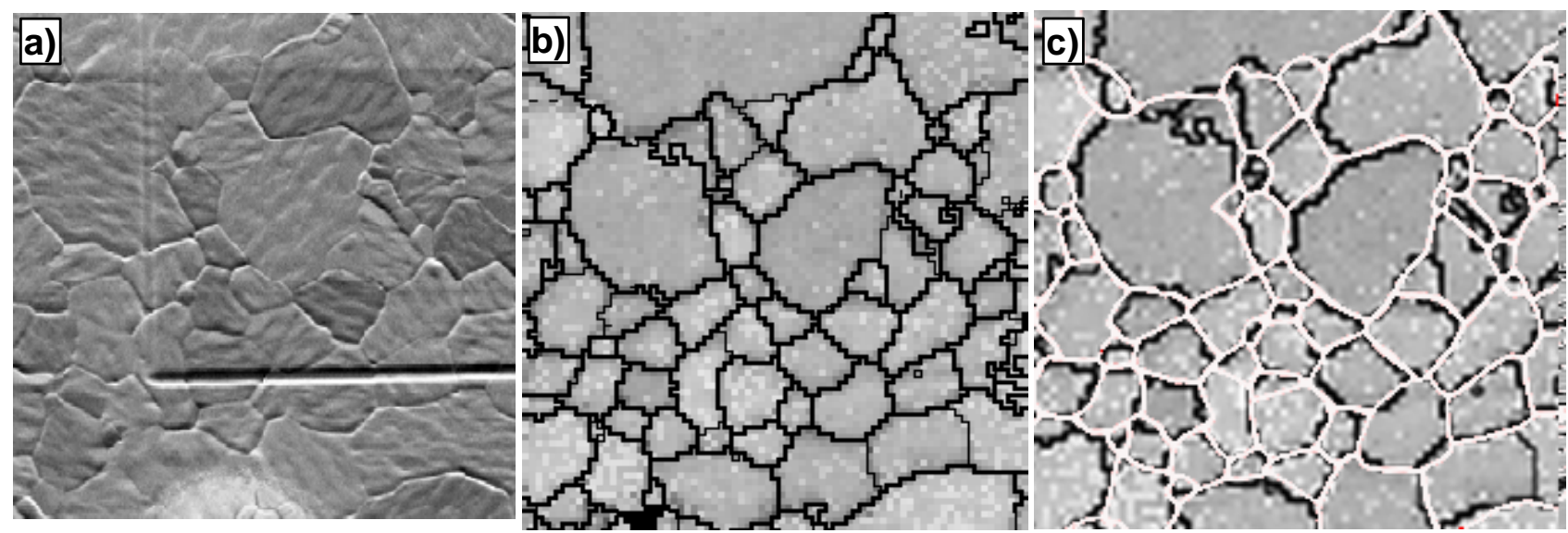

FIG. 3: Direct comparison of FIB imaging and EBSP analysis in same region for sample 2N: a) FIB image of the box corner, b) EBSP orientation image of the same corner, and c) overlay image of FIB traced grains on EBSP image. 